\title{
Generating series of classes of Hilbert schemes of points on orbifolds *
}

\author{
S.M. Gusein-Zade ${ }^{\dagger} \quad$ I. Luengo $\quad$ A. Melle-Hernández ${ }^{\ddagger}$
}

Let $K_{0}\left(\mathcal{V}_{\mathbb{C}}\right)$ be the Grothendieck ring of complex quasi-projective varieties. This is the abelian group generated by isomorphism classes $[X]$ of such varieties modulo the relation $[X]=[X-Y]+[Y]$ for a Zariski closed subvariety $Y \subset X$; the multiplication is defined by the Cartesian product: $\left[X_{1}\right] \cdot\left[X_{2}\right]=\left[X_{1} \times X_{2}\right]$.

Let $\operatorname{Hilb}_{X}^{n}, n \geq 1$, be the Hilbert scheme of zero-dimensional subschemes of length $n$ of a complex quasi-projective variety $X$. According to [4, Hilb ${ }_{X}^{n}$ is a quasi-projective variety. For a point $x \in X$, let Hilb ${ }_{X, x}^{n}$ be the Hilbert scheme of subschemes of $X$ supported at the point $x$.

Let

$$
\begin{aligned}
& \mathbb{H}_{X}(T):=1+\sum_{n=1}^{\infty}\left[\operatorname{Hilb}_{X}^{n}\right] T^{n} \in 1+T K_{0}\left(\mathcal{V}_{\mathbb{C}}\right)[[T]], \quad \text { and } \\
& \mathbb{H}_{X, x}(T):=1+\sum_{n=1}^{\infty}\left[\operatorname{Hilb}_{X, x}^{n}\right] T^{n} \in 1+T K_{0}\left(\mathcal{V}_{\mathbb{C}}\right)[[T]]
\end{aligned}
$$

be the generating series of classes of Hilbert schemes $\operatorname{Hilb}_{X}^{n}$ and $\operatorname{Hilb}_{X, x}^{n}$ in the Grothendieck ring $K_{0}\left(\mathcal{V}_{\mathbb{C}}\right)$.

In [5], there was defined a notion of a power structure over a ring. A power structure over a commutative ring $R$ is a method to give sense to expressions

*Math. Subject Class.: 14C05, 14G10

${ }^{\dagger}$ Partially supported by the grants RFBR-007-00593, INTAS-05-7805 and NWO-RFBR 047.011.2004.026. Address: Moscow State University, Faculty of Mathematics and Mechanics, Moscow, 119991, Russia. E-mail: sabir@mccme.ru

$\ddagger$ The last two authors were partially supported by the grant MTM2007-67908-C02-02. Address: University Complutense de Madrid, Dept. of Algebra, Madrid, 28040, Spain. E-mail: iluengo@mat.ucm.es, amelle@mat.ucm.es 
of the form $\left(1+a_{1} T+a_{2} T^{2}+\ldots\right)^{m}$, where $a_{i}$ and $m$ are elements of the ring $R$. In other words, a power structure is defined by a map $(1+T \cdot R[[T]]) \times R \rightarrow$ $1+T \cdot R[[T]]$ :

$$
(A(T), m) \mapsto(A(T))^{m},\left(A(T)=1+a_{1} T+a_{2} T^{2}+\ldots, a_{i} \in R, m \in R\right),
$$

such that all usual properties of the exponential function hold. In [5], there was described a natural power structure over the Grothendieck ring $K_{0}\left(\mathcal{V}_{\mathbb{C}}\right)$ of complex quasi-projective varieties. It is closely connected with the $\lambda$ structure (see e.g. [9]) on the Grothendieck ring $K_{0}\left(\mathcal{V}_{\mathbb{C}}\right)$ of quasi-projective varieties defined the Kapranov zeta function ([8]). The geometric description of this power structure is given as follows: if $A_{1}, A_{2}, \ldots, M$ are quasiprojective varieties, then the coefficient at $T^{n}$ in the series

$$
\left(1+\left[A_{1}\right] T+\left[A_{2}\right] T^{2}+\ldots\right)^{[M]}
$$

is represented by the configuration space of pairs $(K, \varphi)$ consisting of a finite subset $K$ of the variety $M$ and a map $\varphi$ from $K$ to the disjoint union $\coprod_{i=1}^{\infty} A_{i}$ of the varieties $A_{i}$, such that $\sum_{x \in K} I(\varphi(x))=n$, where $I: \coprod_{i=1}^{\infty} A_{i} \rightarrow \mathbb{Z}$ the tautological function sending the component $A_{i}$ to $i$.

There are two natural homomorphisms from the Grothendieck ring $K_{0}\left(\mathcal{V}_{\mathbb{C}}\right)$ to the ring $\mathbb{Z}$ of integers and to the ring $\mathbb{Z}[u, v]$ of polynomials in two variables: the Euler characteristic (with compact support) $\chi: K_{0}\left(\mathcal{V}_{\mathbb{C}}\right) \rightarrow \mathbb{Z}$ and the Hodge-Deligne polynomial $e: K_{0}\left(\mathcal{V}_{\mathbb{C}}\right) \rightarrow \mathbb{Z}[u, v]: e(X)(u, v)=$ $\sum e^{p, q}(X) u^{p} v^{q}$. These homomorphisms respect the power structures on the correspondings rings (see e.g. [7]).

For a smooth quasi-projective variety $X$ of dimension $d$, the following equation holds in $K_{0}\left(\mathcal{V}_{\mathbb{C}}\right)[[T]]$ :

$$
\mathbb{H}_{X}(T)=\left(\mathbb{H}_{\mathbb{A}^{d}, 0}(T)\right)^{[X]}
$$

([6]), where $\mathbb{A}^{d}$ is the complex affine space of dimension $d$. For $d=2$ in other terms this was proved in the Grothendieck ring of motives by L. Göttsche [3]. For arbitrary $d$, the reduction of the equation (1) under the Hodge-Deligne polynomial homomorphism $e: K_{0}\left(\mathcal{V}_{\mathbb{C}}\right) \rightarrow \mathbb{Z}[u, v]$ was proved by J.Cheah in [1].

Since, for a point $x$ of a smooth variety $X$ of dimension $d$, the Hilbert scheme $\mathrm{Hilb}_{X, x}^{n}$ can be identified with the Hilbert scheme $\mathrm{Hilb}_{\mathbb{A}^{d}, 0}^{n}$, the equation (1) may be written as an integral with respect to the universal Euler characteristic $\chi_{g}(Y)=[Y] \in K_{0}\left(\mathcal{V}_{\mathbb{C}}\right)$ as follows 


$$
\mathbb{H}_{X}(T):=\int_{X} \mathbb{H}_{X, x}(T)^{d \chi_{g}}
$$

Here $d \chi_{g}$ is put in the exponent since the group operation in $1+T \cdot K_{0}\left(\mathcal{V}_{\mathbb{C}}\right)[[T]]$ is the multiplication. Let $\psi$ be a constructible function on a quasi-projective variety $X$ with values in the abelian group $1+T \cdot K_{0}\left(\mathcal{V}_{\mathbb{C}}\right)[[T]]$. If $\psi$ is constant and equal to $\psi_{\Sigma}(T)$ on a stratum $\Sigma$ from a stratification $\mathcal{S}=\{\Sigma\}$ of $X$, then

$$
\int_{X} \mathbb{H}_{X, x}(T)^{d \chi_{g}}=\prod_{\Sigma \in \mathcal{S}}\left(\psi_{\Sigma}(T)\right)^{[\Sigma]} .
$$

If $X$ is not smooth but has isolated singularities, one can easlily see that the equation (2) holds. However this colud not be the case in general. Even the function $\mathbb{H}_{X, x}(T)$ (with values in $\left.1+T \cdot K_{0}\left(\mathcal{V}_{\mathbb{C}}\right)[[T]]\right)$ could be not constructible: singularities of the space $X$ at points of some strata may have moduli. It is interesting to understand to which extend the equation (2) holds for varieties $X$ such that the function $\mathbb{H}_{X, x}(T)$ is constructible.

The described problem does not take place for orbifolds: they have finitely many local models. Here we prove the equation (2) for complex (algebraic) orbifolds.

A $d$-dimensional orbifold $X$ is a complex quasi-projective variety with an atlas of uniformizing systems for Zariski open sets in $X$ (see e.g. [10])). For a Zariski open subset $U \subset X$, an uniformizing system is a triple $(\widetilde{U}, G, \varphi)$, where $G$ is a finite group (depending on $U$ ), $\widetilde{U}$ is a smooth complex $d$ dimensional variety with a $G$-action, and $\varphi$ is an isomorphism $\widetilde{U} / G \rightarrow U$ of varieties (considered with the reduced structures).

For a point $x \in X$, let $(\widetilde{U}, G, \varphi)$ be an uniformizing system for a Zariski open neighbourhood $U$ of the point $x$. Let $\pi_{\widetilde{U}}$ be the natural map $\widetilde{U} \rightarrow \widetilde{U} / G$ and let $\widetilde{x} \in\left(\varphi \circ \pi_{\widetilde{U}}\right)^{-1}(x)$ be a representative of the corresponding orbit. The isotropy group $G_{\widetilde{x}}=\{g \in G: g \widetilde{x}=\widetilde{x}\}$ of the point $\widetilde{x}$ acts on the tangent space $T_{\widetilde{x}} \widetilde{U}$ by a representation $\alpha=\alpha_{\widetilde{x}}: G_{\widetilde{x}} \rightarrow G L(d, \mathbb{C})$. Moreover, there exists a system of local parameters $z_{1}, \ldots, z_{d}$ at the point $\widetilde{x}$ such that the action of the group $G_{\widetilde{x}}$ on the manifold $\widetilde{U}$ is given by standard linear equations corresponding to the representation:

$$
g^{*} z_{i}=\sum_{j=1}^{d} \alpha_{i, j}(g) z_{j}
$$


where $\left(\alpha_{i, j}(g)\right)=\alpha(g)$.

One has: 1) the Hilbert scheme $\operatorname{Hilb}_{X, x}^{n}$ of 0 -dimensional subschemes on $X$ supported at the point $x$ is isomorphic to the Hilbert scheme $\left.\operatorname{Hilb}_{\mathbb{A}^{d} / G_{\widetilde{x}}, 0}^{n} ; 2\right)$ the partitioning of $U$ into parts corresponding to different conjugacy classes of isotropy subgroups $G_{\widetilde{x}} \subset G$ and to different (non-isomorphic) representations of the group $G_{\widetilde{x}}$ is a stratification of $U$. Therefore $\mathbb{H}_{X, x}(T)$ is a constructible function on $X$ with values in $1+T \cdot K_{0}\left(\mathcal{V}_{\mathbb{C}}\right)[[T]]$.

Theorem 1 For an orbifold $X$, the following equation holds in $1+T K_{0}\left(\mathcal{V}_{\mathbb{C}}\right)[[T]]$ :

$$
\mathbb{H}_{X}(T)=\int_{X} \mathbb{H}_{X, x}(T)^{d \chi_{g}} .
$$

Proof. For a locally closed subvariety $Y \subset X$, let $\operatorname{Hilb}_{X, Y}^{n}$ be the Hilbert scheme of subschemes of length $n$ of $X$ supported at points of $Y$ and let

$$
\mathbb{H}_{X, Y}(T):=1+\sum_{n=1}^{\infty}\left[\operatorname{Hilb}_{X, Y}^{n}\right] T^{k}
$$

be the corresponding generating series. If $Y$ is a Zariski closed subset of $X$, then

$$
\mathbb{H}_{X}(T)=\mathbb{H}_{X, Y}(T) \cdot \mathbb{H}_{X, X \backslash Y}(T) .
$$

Therefore it is sufficient to prove the equation (2) when $X$ is covered by one uniformizing system $(\widetilde{U}, G, \varphi)$ such that $\widetilde{U}$ lies in an affine space $\mathbb{A}^{N}$.

Let us fix a subgroup $G^{\prime}$ of the group $G$ and a representation $\alpha: G^{\prime} \longrightarrow$ $G L(d, \mathbb{C})$. Let $X_{G^{\prime}, \alpha}$ be the image under $\varphi \circ \pi_{G}$ of the set of points $\widetilde{x} \in \widetilde{U}$ such that $G_{\widetilde{x}}=G^{\prime}$ and the representation of the group $G^{\prime}$ on the tangent space $T_{\widetilde{x}} \widetilde{U}$ is isomorphic to $\alpha$. Using (6) again, one can see that it is sufficient to prove the formula

$$
\mathbb{H}_{X, Y}(T)=\left(\mathbb{H}_{\mathbb{A}^{d} /\left(G^{\prime}, \alpha\right), 0}(T)\right)^{[Y]}
$$

for any point $x \in X_{G^{\prime}, \alpha}$ and for a certain Zariski open neighbourhood $Y$ of this point in an irreducible component of $X_{G^{\prime}, \alpha}$.

Let $\widetilde{x} \in\left(\varphi \circ \pi_{\widetilde{U}}\right)^{-1}(x)$ be a point in $\widetilde{U}$ such that $G_{\widetilde{x}}=G^{\prime}$. Then the representation of $G^{\prime}$ on the tangent space $T_{\widetilde{x}} \widetilde{U}$ is automatically isomorphic to $\alpha$. Let $u_{1}, \ldots, u_{d}$ be a regular system of parameters at the point $\widetilde{x}$ (for 
example one may suppose that $\widetilde{x}=0$ and $u_{1}, \ldots, u_{d}$ are $d$ of the standard coordinates on $\mathbb{A}^{N}$ such that the projection of the tangent space $T_{\widetilde{x}} \widetilde{U}$ to the corresponding $d$-dimensional coordinate plane is non-degenerate (i.e. $u_{1}-$ $u_{1}^{0}, \ldots, u_{d}-u_{d}^{0}$ is a regular system of parameters at each point from a Zariski open neighbourhood of $\widetilde{x}$ ).

Let us suppose that the parameters $u_{1}, \ldots, u_{d}$ are chosen in such a way that, in the corresponding coordinates on the tangent space $T_{\widetilde{x}} \widetilde{U}$, the action of the group $G^{\prime}$ is given by the standard equations (4). Define a new regular system of parameters $\widetilde{u}_{1}, \ldots, \widetilde{u}_{d}$ at the point $\widetilde{x}$ by the equations

$$
\widetilde{u}_{i}=\frac{1}{\left|G^{\prime}\right|} \sum_{g \in G^{\prime}} \sum_{j} \alpha_{i, j}\left(g^{-1}\right) g^{*} u_{j} .
$$

One has

$$
g^{*} \widetilde{u}_{i}=\sum_{j} \alpha_{i, j}(g) \widetilde{u}_{j}
$$

Therefore, $\widetilde{u}_{1}-\widetilde{u}_{1}^{0}, \ldots, \widetilde{u}_{d}-\widetilde{u}_{d}^{0}$ is a regular system of parameters at each point from a Zariski open neighbourhood of $\widetilde{x}$ in the corresponding irreducible component of $\left(\varphi \circ \pi_{G}\right)^{-1} X_{G^{\prime}, \alpha}$. It defines a map $\underline{\widetilde{u}}-\underline{\widetilde{u}}^{0}: \widetilde{U}, \widetilde{x}^{\prime} \rightarrow \mathbb{A}^{d}, 0$. There is a commutative diagram

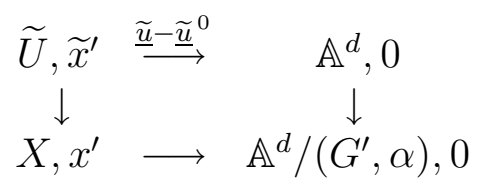

and the lower map identifies the Hilbert scheme of points on $X$ supported at the point $x^{\prime}$ with the Hilbert scheme of points on $\mathbb{A}^{d} /\left(G^{\prime}, \alpha\right)$ supported at the origin.

This way, a zero-dimensional subscheme on $X$ supported at poins of $Y$ is defined by a finite subset $K \subset Y$ to each point $x$ of which there corresponds a zero-dimensional subscheme on $\mathbb{A}^{d} /\left(G^{\prime}, \alpha\right)$ supported at the origin. The length of the subscheme is equal to the sum of lengths of the corresponding subschemes of $\mathbb{A}^{d} /\left(G^{\prime}, \alpha\right)$. As it follows from the geometric description of the power structure over the Grothendieck ring of quasi-projective varieties, the coefficient at $T^{n}$ in the right hand side of the equation (7) is represented just by the configuration space of such objects. This proves the statement.

Reductions of the equation (21) under the Euler characteristic homomorphism and the Hodge-Deligne polynomial homomorphism gives equations for 
the generating series of the corresponding invariants of Hilbert schemes of points on orbifolds. In particular

$$
1+\sum_{n=1}^{\infty} \chi\left(\operatorname{Hilb}_{X}^{n}\right) T^{n}=\int_{X}\left(1+\sum_{n=1}^{\infty} \chi\left(\operatorname{Hilb}_{X, x}^{n}\right) T^{n}\right)^{d \chi} .
$$

In [2], J. Cheah considered nested Hilbert schemes on a smooth $d$-dimensional complex quasi-projective variety $X$. For $\underline{n}=\left(n_{1}, \ldots, n_{r}\right) \in \mathbb{Z}_{>0}^{r}$, the nested Hilbert scheme $Z \frac{n}{X}$ of depth $r$ is the scheme which parametrizes collections of the form $\left(Z_{1}, \ldots, Z_{r}\right)$, where $Z_{i} \in$ Hilb $_{X}^{n_{i}}$ and $Z_{i}$ is a subscheme of $Z_{j}$ for $i<j$. The scheme $Z \frac{n}{X}$ is non-empty only if $n_{1} \leq n_{2} \leq \ldots \leq n_{r}$; notice that $Z_{X}^{(n)}=\operatorname{Hilb}_{X}^{n} \cong Z_{X}^{(n, \ldots, n)}$.

For $Y \subset X$, let $Z_{X, Y}^{n}$ be the scheme which parametrizes collections $\left(Z_{1}, \ldots, Z_{r}\right)$ from $Z_{X}^{n}$ with $\operatorname{supp} Z_{i} \subset Y$. For $Y=\{x\}, x \in X$, we shall use the notation $Z \frac{n}{X}, x$.

For $r \geq 1$, let $\underline{T}=\left(T_{1}, \ldots, T_{r}\right)$ and

$$
\mathcal{Z}_{X}^{(r)}(\underline{T}):=1+\sum_{\underline{n} \in \mathbb{Z}_{\geq 0}^{r} \backslash\{0\}}\left[Z^{\frac{n}{X}}\right] \underline{T}^{\underline{n}}, \quad \mathcal{Z}_{X, x}^{(r)}(\underline{T}):=1+\sum_{\underline{n} \in \mathbb{Z}_{\geq 0}^{r} \backslash\{0\}}\left[Z_{\bar{X}, x}^{n}\right] \underline{T}^{\underline{n}},
$$

be the generating series of classes of the nested Hilbert schemes $Z \frac{n}{X}$ of depth $r$ (resp. of those supported at the point $x$ ).

A series $A(\underline{T})=1+\sum_{\underline{n} \in \mathbb{Z}_{\geq 0}^{r} \backslash\{0\}} a_{\underline{n}} \underline{T}^{\underline{n}}, \underline{T}^{\underline{n}}:=T_{1}^{n_{1}} \cdot \ldots \cdot T_{r}^{n_{r}}, a_{\underline{n}} \in K_{0}\left(\mathcal{V}_{\mathbb{C}}\right)$, has a unique representation of the form $A(\underline{T})=\prod_{\underline{k} \in \mathbb{Z}_{\geq 0}^{r} \backslash\{0\}}\left(1-\underline{T}^{\underline{k}}\right)^{-s_{\underline{k}}}, s_{\underline{k}} \in K_{0}\left(\mathcal{V}_{\mathbb{C}}\right)$. Then for $m \in K_{0}\left(\mathcal{V}_{\mathbb{C}}\right), A(\underline{T})^{m}:=\prod_{\underline{k} \in \mathbb{Z}_{\geq 0}^{r} \backslash\{0\}}\left(1-\underline{T}^{\underline{k}}\right)^{-m s_{\underline{k}}}$ (see [7]).

For a smooth quasi-projective variety $X$ of dimension $d$, the following equation holds in $\left.K_{0}\left(\mathcal{V}_{\mathbb{C}}\right)[[\underline{T}]]\right)$ :

$$
\mathcal{Z}_{X}^{(r)}(\underline{T})=\left(\mathcal{Z}_{\mathbb{A}^{d}, 0}^{(r)}(\underline{T})\right)^{[X]}
$$

([7]).

Using the same arguments as in the proof of Theorem 1, one gets the following statetment

Theorem 2 For an orbifold $X$, the following equation holds

$$
\mathcal{Z}_{X}^{(r)}(\underline{T})=\int_{X}\left(\mathcal{Z}_{X, x}^{(r)}(\underline{T})\right)^{d \chi_{g}}
$$




\section{References}

[1] J. Cheah, On the cohomology of Hilbert schemes of points. J. Algebraic Geom. 5 (1996), no.3, 479-511.

[2] J. Cheah, The virtual Hodge polynomials of nested Hilbert schemes and related varieties. Math. Z. 227 (1998), 479-504.

[3] L. Göttsche, On the motive of the Hilbert scheme of points on a surface. Math. Res. Lett. 8 (2001), no.5-6, 613-627.

[4] A. Grothendieck, Techniques de construction et théorèmes d'existence en géométrie algébrique. IV. Les schémas de Hilbert. Séminaire Bourbaki, Vol. 6, Exp. No. 221, 249-276, Soc. Math. France, Paris, 1995.

[5] S.M. Gusein-Zade, I. Luengo and A. Melle-Hernández, A power structure over the Grothendieck ring of varieties. Math. Res. Lett. 11 (2004), no.1, 49-57.

[6] S.M. Gusein-Zade, I. Luengo and A. Melle-Hernández, Power structure over the Grothendieck ring of varieties and generating series of Hilbert schemes of points. Michigan Mathematical Journal, 2006, v.54, 353-359.

[7] S.M. Gusein-Zade, I. Luengo and A. Melle-Hernández, On the power structure over the Grothendieck ring of varieties and its applications. Proceedings of the Steklov Institute of Mathematics, 2007, v.258, 5364.

[8] M. Kapranov, The elliptic curve in the S-duality theory and Eisenstein series for Kac-Moody groups. Preprint, ArXiv math.AG/0001005.

[9] D. Knutson, $\lambda$-rings and the representation theory of the symmetric group. Lecture Notes in Mathematics, Vol. 308. Springer-Verlag, BerlinNew York, 1973.

[10] I. Moerdijk, D.A. Pronk, Orbifolds, sheaves and groupoids. K-Theory 12 (1997), no.1, 3-21. 